\title{
Early postoperative HPA-axis testing after pituitary tumor surgery: reliability and safety of basal cortisol and CRH test
}

\author{
Friso de Vries $\mathbb{1}^{1,2} \cdot$ Daniel J. Lobatto $\mathbb{1}^{2,3} \cdot$ Leontine E. H. Bakker ${ }^{1,2} \cdot$ Wouter R. van Furth $\mathbb{1}^{2,3}$. \\ Nienke R. Biermasz $\mathbb{I D}^{1,2}$. Alberto M. Pereira $\mathbb{D D}^{1,2}$
}

Received: 11 June 2019 / Accepted: 13 September 2019 / Published online: 25 September 2019

(c) The Author(s) 2019

\begin{abstract}
Purpose To assess the reliability and safety of a postsurgical evaluation strategy of adrenal function using $\mathrm{CRH}$ stimulation and basal cortisol concentrations after transsphenoidal pituitary surgery.

Methods Retrospective cohort study of all patients undergoing endoscopic transsphenoidal surgery from 2010 to 2017 , in whom early postoperative basal cortisol and/or CRH-stimulated cortisol secretion were available, including confirmation of adrenal function during follow-up. Patients with Cushing's disease were excluded. Optimal test performances were assessed using ROC analysis.

Results A total of 156 patients were included. Sensitivity and specificity of the CRH test were $78 \%$ and $90 \%$, respectively, and $86 \%$ and $92 \%$ for basal cortisol, respectively, using an optimal cutoff of $220 \mathrm{nmol} / \mathrm{L}$. Eight patients had false-negative test results with the CRH test (normal test but adrenal insufficient at follow-up), and six patients with basal cortisol, the majority of which had multiple pituitary hormone deficiencies and fluid imbalances. No clinical adverse events occurred in patients with false-negative test results. The diagnostic performance of a single basal cortisol measurement was superior to the CRH test.

Conclusions The early postoperative basal cortisol is a safe and simple measurement to guide (dis)continuation of hydrocortisone replacement. However, disturbing factors, e.g., sodium balance disorders, contraceptives, untreated hypopituitarism, and illness impact the interpretation and in those cases this measure is unreliable. We propose an algorithm in which hydrocortisone replacement at discharge is based on basal cortisol $<220 \mathrm{nmol} / \mathrm{L}$ on postoperative day 2 or 3 in a stable condition.
\end{abstract}

Keywords Adrenal insufficiency $\cdot \mathrm{HPA}$-axis $\cdot$ Cortisol $\cdot$ Transsphenoidal surgery $\cdot$ Pituitary $\cdot$ Postoperative testing

\section{Introduction}

Transsphenoidal endoscopic surgery is the cornerstone of treatment for the majority of patients with pituitary tumors. A possible complication of surgery is the onset of new pituitary insufficiencies. This may eventually recover in

Friso de Vries

f.de_vries@lumc.nl

1 Department of Medicine, Division of Endocrinology, Leiden University Medical Centre, Leiden, The Netherlands

2 Center for Endocrine Tumors Leiden (CETL), Center for Pituitary Care, Leiden University Medical Center, Leiden, The Netherlands

3 Department of Neurosurgery, Leiden University Medical Centre, Leiden, The Netherlands some, but not in all patients. Correct interpretation of adrenal function early after surgery is of paramount importance. Cortisol deficiency may be life-threatening and unnecessary glucocorticoid replacement can be harmful and may cause (long-lasting) side effects. A meticulous postsurgical assessment of adrenal function is therefore mandatory. However, there is no consensus on how to evaluate adrenal function directly after surgery.

Several tests are available for the assessment of adrenal function, of which the insulin tolerance test (ITT) is considered as the gold standard. The ITT, however, is not suitable for the immediate postoperative period, as it is burdensome, and has contraindications. The ACTH test may not detect cases of new-onset secondary adrenal insufficiency (AI). Alternatives are the CRH-stimulation test, the metyrapone test, and measurements of nonstimulated basal cortisol, or random serum cortisol 
Fig. 1 Flowchart of patient selection

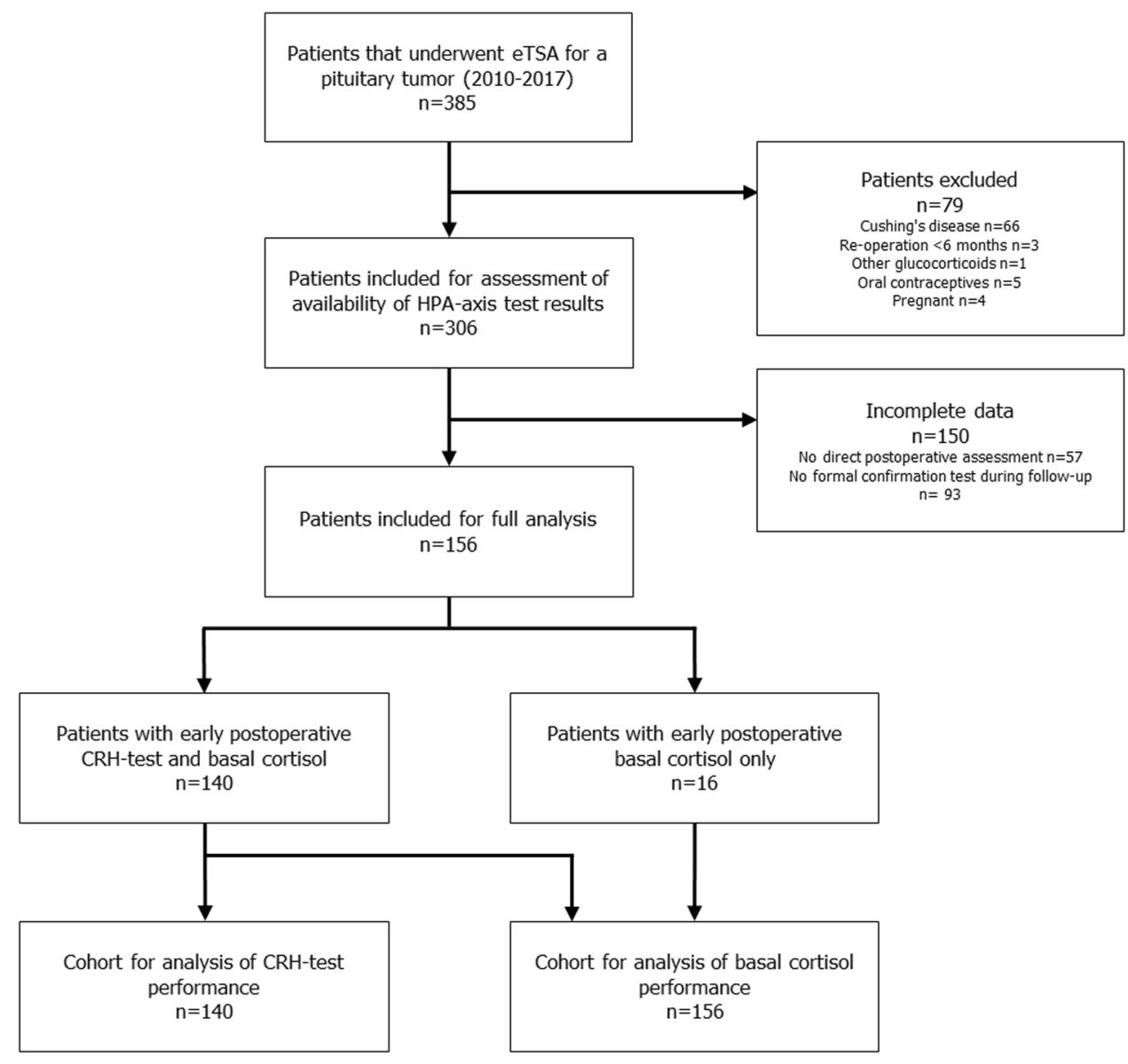

concentrations [1-5]. Available data assessing the safety and accuracy of these tests are limited [2, 3, 6-12]. Moreover, a comparison between all different assessment methods in the postoperative setting has not yet been performed.

The Center for Endocrine Tumors Leiden is a tertiary referral center and coordinating center of the European Reference Network for Rare Endocrine Conditions (EndoERN, www.endo-ern.eu). At our Reference Centre for pituitary care, a CRH-stimulation test $\sim 5$ days after surgery has been the preferred test to evaluate adrenal function after surgery since 1990 . We previously reported on the clinical applicability and safety of the test in our cohort of patients treated between 1990 and 2009. This study stated that the strategy to continue hydrocortisone replacement guided by the $\mathrm{CRH}$ test appeared to be safe and did not result in any case of adrenal crises [4]. Although safe, the early postoperative CRH test could not reliably predict long-term adrenal function. Therefore, retesting was considered mandatory. Nowadays, the introduction of endoscopic surgery and lower perioperative hydrocortisone replacement doses allow earlier discharge in a significant proportion of patients. This prompted us to critically re-evaluate our postsurgical evaluation strategy of adrenal function.
The aim of the present study was to re-assess the current practice by studying performance and clinical safety of the $\mathrm{CRH}$ test directly after surgery in diagnosing AI as compared with a confirmation test during follow-up. Next, we assessed the reliability and safety of a simplified protocol based on a postoperative single basal cortisol measurement only. Performances of both tests were compared in order to optimize the postsurgical evaluation of adrenal function.

\section{Methods}

\section{Patient selection}

(Fig. 1) We performed a retrospective chart review of all consecutive patients who underwent endoscopic transsphenoidal pituitary surgery at our center between January 2010 and December $2017(n=385)$. Patients were excluded in case of Cushing's disease $(n=66)$, re-operation within 6 months $(n=3)$, treatment with glucocorticoids other than hydrocortisone $(n=1)$, use of oral contraceptives $(n=5)$, and pregnancy $(n=4)$. Patients with available data on early postoperative testing (basal cortisol concentrations and/or $\mathrm{CRH}$ test) and a conclusive confirmation test during follow-up 
were eligible for inclusion. Early postoperative testing was defined as a maximum of 2 weeks after surgery. A conclusive confirmation test was defined as a cortisol response after stimulation with either CRH or ITT or a basal cortisol below or above the reference range. Basal cortisol values within the reference ranges in the absence of a stimulation test were considered uninterpretable and these patients were excluded. In 57 patients, no direct postoperative evaluation of residual cortisol secretion was performed and in 93 patients no formal confirmation test was performed during follow-up. This resulted in the inclusion of 156 patients (40.5\% of the full cohort). In 16 patients $(10.3 \%$ ) results of early basal cortisol were available, but not of an early $\mathrm{CRH}$ test.

\section{Management regarding hydrocortisone replacement}

On the day of surgery, patients received $50 \mathrm{mg} / 24 \mathrm{~h}$ intravenous hydrocortisone continuously. On postoperative day 1 this was switched to a $20-10-10 \mathrm{mg}$ oral dose, and from postoperative day 2 onwards 10-5-5 mg hydrocortisone was administered. Five days after surgery, or later depending on the clinical situation, a CRH test was performed. Hydrocortisone was withdrawn prior to discharge in case of a normal cortisol response. Hydrocortisone was continued in case of an abnormal peak cortisol at least until the patient was retested (usually within the first 6 months). Patients with very low cortisol levels and evident hypopituitarism were not retested, patients with high cortisol responses after CRH-stimulation and no other signs of hypopituitarism were not routinely retested, based on the clinicians' decision. All patients were closely monitored and adrenal function was re-evaluated in case of clinical suspicion of possible AI.

\section{Endocrine assessment}

\section{CRH test}

Patients were instructed to take the last hydrocortisone dose at least $18 \mathrm{~h}$ prior to the test. After an overnight fast, $100 \mu \mathrm{g}$ of corticoliberine (human CRH) (Ferring Pharmaceuticals, Hoofddorp, the Netherlands) was administered intravenously, and blood samples were collected for measurement of cortisol and ACTH at $-15,-5,15,30,45$, and $60 \mathrm{~min}$ after CRH administration. Peak cortisol of $>430 \mathrm{nmol}$ and a peak ACTH of $>40 \mathrm{ng} / \mathrm{L}$ indicated a normal response to CRH stimulation [13, 14].

\section{Basal cortisol level}

Blood samples were taken between 6 and 8 A.M. after withdrawing hydrocortisone for at least $18 \mathrm{~h}$. Cortisol concentrations $<70 \mathrm{nmol} / \mathrm{L}$ were considered as evidence of AI. Cortisol concentrations of $>430 \mathrm{nmol} / \mathrm{L}$ as evidence of normal HPA-axis functioning. Intermediate values were regarded as inconclusive and indicated further (dynamic) testing.

\section{ITT}

The ITT was performed after an overnight fast after withdrawal of hydrocortisone replacement for at least $18 \mathrm{~h}$. A total of $0.1 \mathrm{U} / \mathrm{kg}$ body weight of intravenous insulin (Novorapid, Novo Nordisk Farma, Bagsvaerd, Denmark) was administered to induce adequate hypoglycemia (defined as nadir glucose $<2.2 \mathrm{mmol} / \mathrm{L}$ in the presence of neuroglycopenic symptoms). Blood samples were drawn for the measurement of cortisol, $\mathrm{ACTH}$, and $\mathrm{GH}$ at $-15,-5$, $15,45,60$, and $90 \mathrm{~min}$ after insulin administration. Peak cortisol of $>430 \mathrm{nmol} / \mathrm{L}$ was defined as a normal response to hypoglycemia.

\section{ACTH test}

The ACTH test was performed after an overnight fast after withdrawal of hydrocortisone replacement for at least $18 \mathrm{~h}$. Tetracosactide $250 \mu \mathrm{g}$ (Synacthen, Novartis, Arnhem, the Netherlands) was administered and blood is drawn for cortisol and ACTH levels at $-15,-5$, and $30 \mathrm{~min}$ after injection of ACTH. A normal cortisol response was defined as peak cortisol $>430 \mathrm{nmol} / \mathrm{L}$. An ACTH test was deemed reliable after at least 12 months after surgery.

Adrenal crises were defined and graded according to the expert opinion paper by B. Allolio [15].

\section{Assays}

Cortisol was measured using electrochemiluminescent immunoassay (ECLIA) on a Cobas 8000 module e602 (Roche Diagnostics, Mannheim, Germany). Up to January 1st, 2016, blood samples were first treated with Elecsys Cortisol I reagent. From January 1st, 2016 onward, the Elecsys Cortisol II reagent was used. The use of the cortisol II reagent results in a factor 0.78 lower cortisol values than compared with the cortisol I reagent. We transformed each result of the cortisol I reagent to a cortisol II result (multiplied by 0.78). ACTH was measured using ECLIA on the same Cobas module and with an ACTH reagent (Roche Diagnostics, Mannheim, Germany). The detection limit ranges from 1 to $2000 \mathrm{ng} / \mathrm{L}$.

\section{Statistical analysis}

Statistical analysis was performed using IBM SPSS statistics 23 (SPSS Inc. Chicago, Illinois, USA). Descriptive 
statistics were used for baseline characteristics, with continuous variables being reported as means with range. Separate analyses were performed for the performances of the CRH test and basal cortisol. Contingency tables were used to calculate the performance indices of both tests. The positive likelihood ratio $(\mathrm{LR}+)$ was defined as sensitivity divided by 1 -specificity. Inversely, the negative LR - was calculated as 1-sensitivity divided by specificity. Receiver operator characteristics (ROC) were performed for determining optimum cut-off values. Youden's index (sensitivity + specificity-1) was used to assess the optimal cutoff. First, the results of early postoperative testing were compared with the result of an ITT during follow-up (gold standard). Because of the low sample size, thereafter the results of early postoperative testing were compared with all confirmation tests during follow-up. The obtained cut-off values were used to assess the diagnostic performance of the tests. Because the obtained cut-off values in this study highly adhere to the cohort and underdiagnosis of AI can be dangerous, we chose cut-off values that are somewhat on the safe side.

\section{Results}

\section{Baseline characteristics}

One hundred and fifty-six patients were included, with the following diagnoses: pituitary adenoma (Cushing's disease excluded): $n=140$ (89.7\%), craniopharyngioma: $n=9$ (5.8\%), and Rathke's cleft cyst: $n=7$ (4.5\%) (Table 1). Mean age was 53.2 years, and $51 \%$ of patients were female. Thirty-four patients (21.8\%) were adrenal insufficient before surgery. Mean follow-up was 4.2 years (range 0.74-8.64 years), with a total of 656 patient-years of follow-up.

\section{ROC analysis of early postoperative CRH test}

Results of early postoperative CRH testing with subsequent ITT during follow-up were available in 61 patients (Fig. 2a, c). The ROC analysis of the early postoperative CRH test with the ITT as reference showed an AUC of 0.767 (95\% CI $0.614-0.920$ ). A peak cortisol level of $574 \mathrm{nmol} / \mathrm{L}$ corresponded with $100 \%$ sensitivity, and a specificity of $46 \%$, whereas a cutoff of $310 \mathrm{nmol} / \mathrm{L}$ corresponded to a specificity of $100 \%$, but with only $22 \%$ sensitivity. The optimum cut-off value was $424 \mathrm{nmol} / \mathrm{L}$, with a Youden index of 0.421 (sensitivity $55.6 \%$ and specificity $86.5 \%$ ).

A ROC analysis was also performed for all different confirmation tests $(n=140)$ which yielded nearly the same optimal cutoff with higher statistical performances: $430 \mathrm{nmol} / \mathrm{L}$. The upper cutoff in this analysis was $672 \mathrm{nmol} / \mathrm{L}$
Table 1 Baseline characteristics

\begin{tabular}{ll}
\hline Baseline characteristics & $\begin{array}{l}\text { Number of patients } \\
(n=156)\end{array}$ \\
\hline M/F & $76 / 80$ \\
Mean age (years) & 53.2 (range 17-85) \\
Diagnosis & \\
Non-functioning adenoma & 98 \\
GH-producing adenoma & 22 \\
Prolactinoma & 20 \\
Other adenoma & 7 \\
Craniopharyngioma & 11 \\
RCC & 8 \\
Preoperative pituitary function & \\
No deficiencies & 80 \\
Preoperative single pituitary deficiency & 26 \\
Preoperative multiple pituitary & 48 \\
deficiencies & \\
Preoperative AI & 34 \\
Not documented & $2^{\mathrm{a}}$ \\
Confirmation test & \\
Single cortisol measurement & 39 \\
ITT & 63 \\
CRH test & 52 \\
ACTH test & 2 \\
\hline
\end{tabular}

${ }^{\mathrm{a}}$ In two patients no preoperative hormonal panel was performed because of immediate surgery following pituitary apoplexy

${ }^{\mathrm{b}}$ During follow-up (mean 8.0 months after surgery)

and the lower cutoff $232 \mathrm{nmol} / \mathrm{L}$. Therefore, a CRHstimulated cortisol concentration of $430 \mathrm{nmol} / \mathrm{L}$ (the already used cutoff in daily practice) was used as the cutoff for test performance. Performance indices for both references are presented in Table 4.

\section{Early postoperative CRH testing versus confirmation test during follow-up ( $n=140$ patients)}

Early postoperative CRH testing showed a mean peak cortisol concentration of $545 \mathrm{nmol} / \mathrm{L}$ (range 10-1211).

A cortisol peak of below $430 \mathrm{nmol} / \mathrm{L}$ was observed in 32 patients $(22.9 \%)$, all of whom continued hydrocortisone replacement at discharge. AI was confirmed in 23 of 32 patients (71.9\%) at follow-up (ITT $n=12$, CRH test $n=$ 12, ACTH test $n=2$, basal cortisol $n=6$ ). Hydrocortisone was discontinued in nine patients with normal test results during follow-up (Table 2: patients 1-9).

The cortisol response to CRH early after surgery was above $430 \mathrm{nmol} / \mathrm{L}$ in 108 patients $(77.1 \%$ ), who discontinued hydrocortisone thereafter. Eight of these (7.4\%) were diagnosed with AI during follow-up (ITT $n=4$, CRH test $n=2$, and basal cortisol $n=2$, see Table 3: patients 

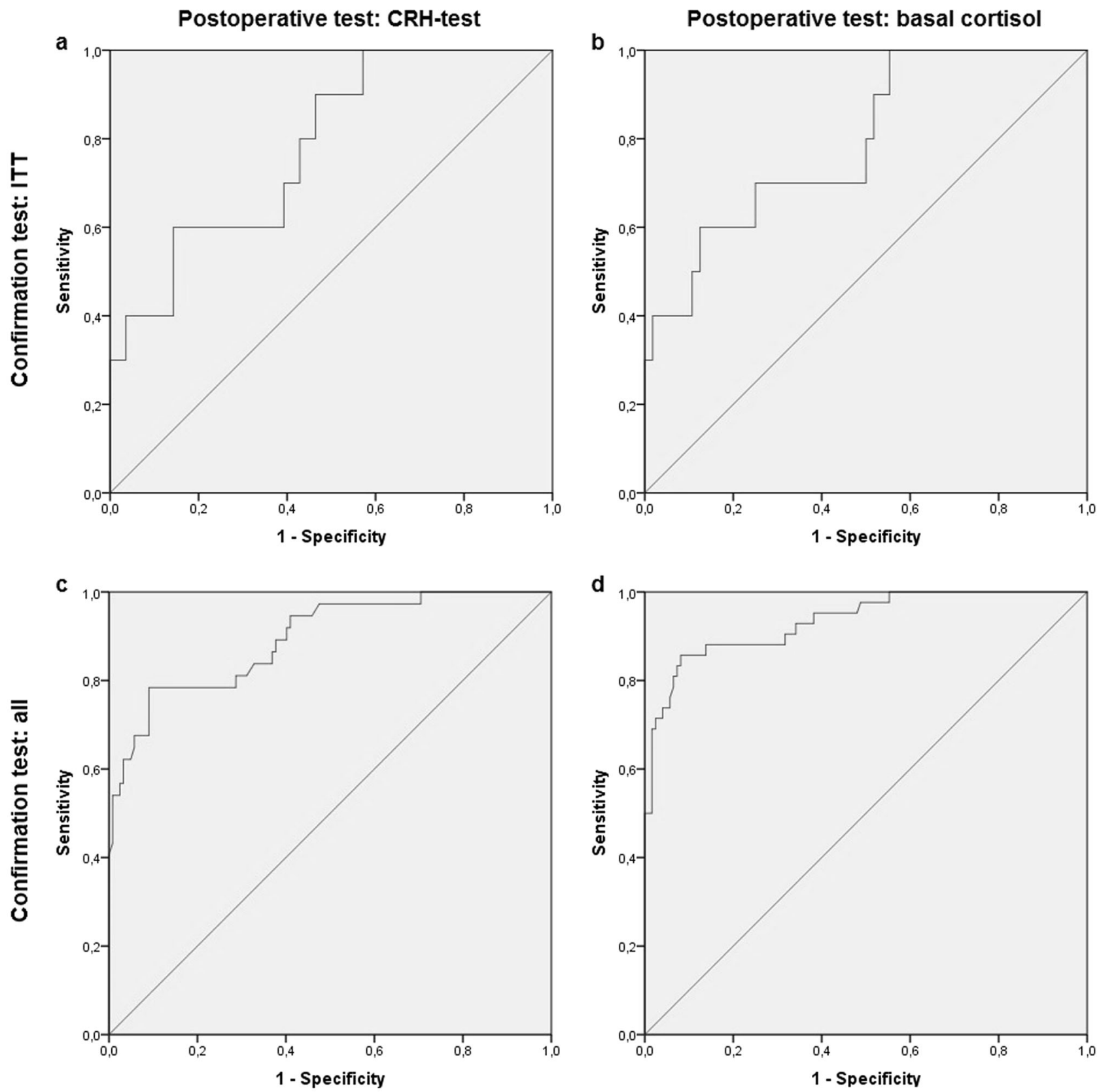

Fig. 2 ROC-curve of CRH test and basal cortisol as confirmed with ITT and all confirmation tests, with reference line (diagonal). ROCcurve of CRH test and basal cortisol as confirmed with ITT and all confirmation tests, with reference line (diagonal). a CRH test vs ITT,

AUC: 0.767 (95\%CI 0.614-0.920), b Basal cortisol vs ITT, AUC: 0.767 (95\%CI 0.608-0.927), c CRH test vs all confirmation tests, AUC: 0.885 (95\%CI 0.817-953), d Basal cortisol vs all confirmation tests, AUC: 0.928 (95\%CI 0.879-976)

1-8). Normal adrenal function was confirmed during follow-up in 100 patients, (ITT $n=45$ ), CRH test $n=35$, and basal cortisol $n=20$ ). As can be appreciated from Table 3, two of these eight patients with a false-negative test result had new-onset hypothyroidism after surgery, and five patients had a concomitant diagnosis of DI or SIADH at the time of testing $(n=4)$ or which manifested early after the test $(n=1)$.

\section{ROC analysis of basal (nonstimulated) cortisol}

Results of early postoperative basal cortisol measures with subsequent ITT during follow-up were available in 63 patients (Fig. 2b, d). The ROC analysis of basal cortisol with the ITT as reference showed an AUC of 0.767 (95\%CI 0.608-0.927). A basal cortisol concentration of $325 \mathrm{nmol} / \mathrm{L}$ corresponded with $100 \%$ sensitivity and a specificity of $42.6 \%$. A cortisol 


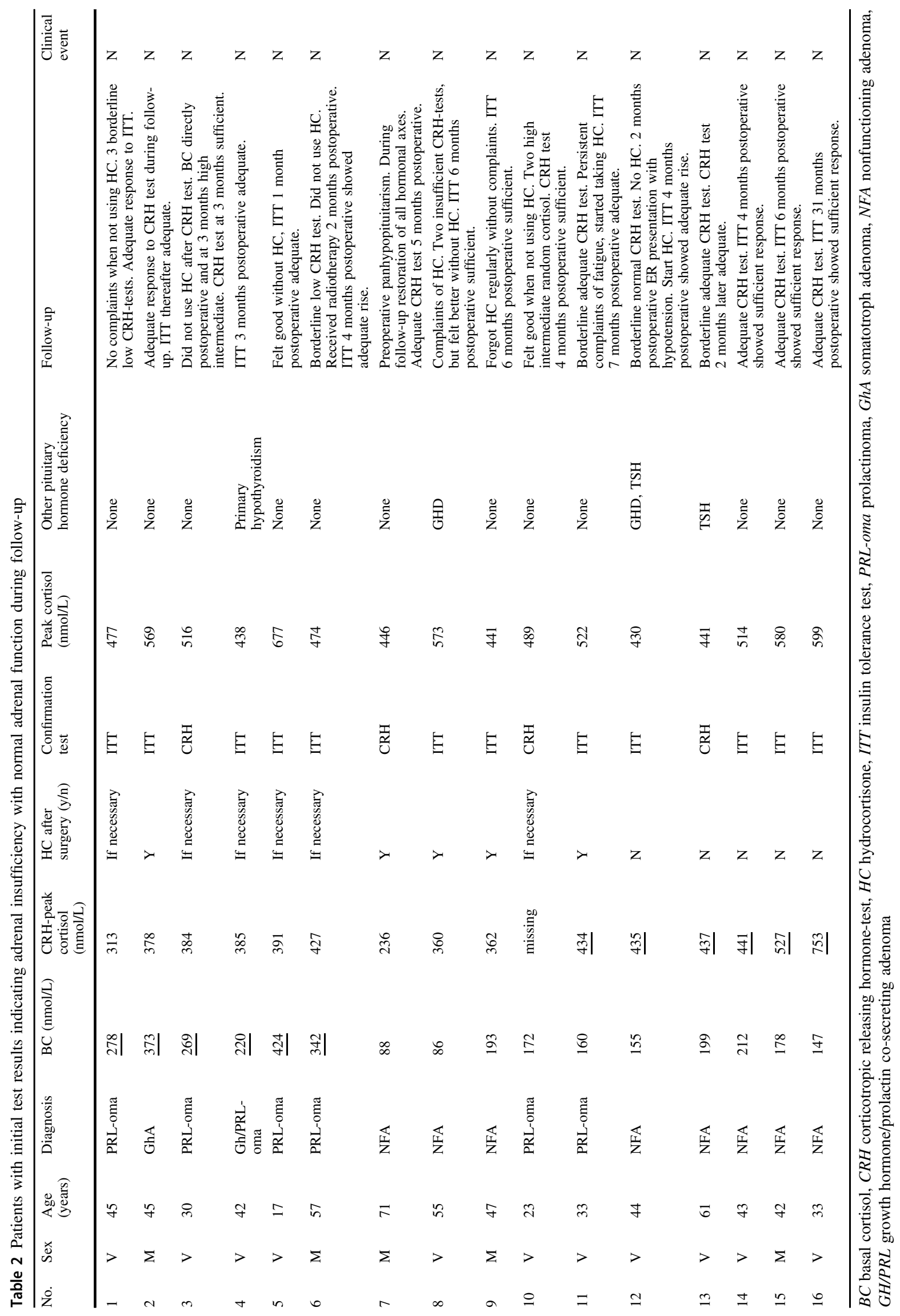




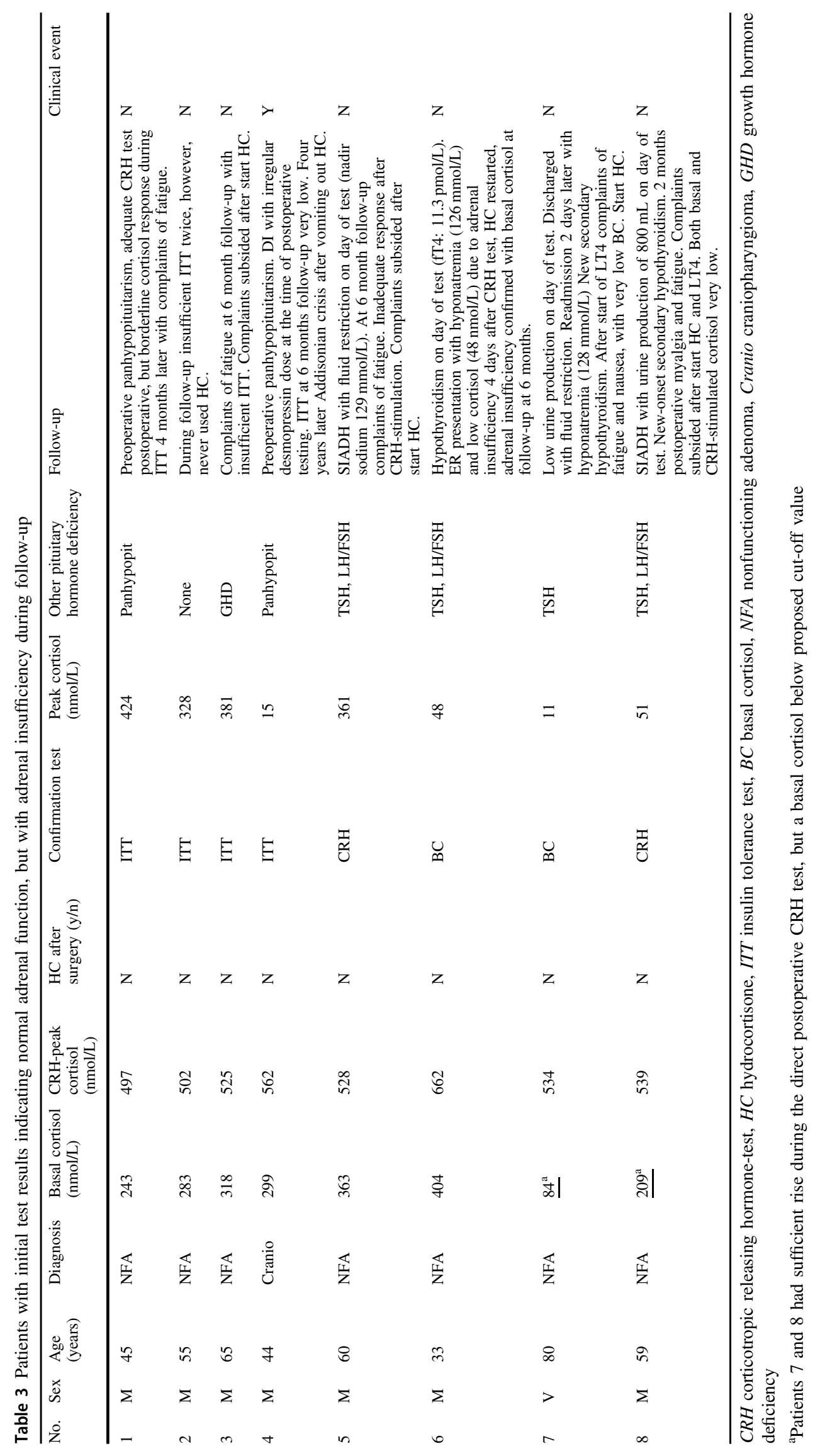


Table 4 Diagnostic performance indices of the early postoperative CRH stimulation test and of a single morning cortisol measurement

\begin{tabular}{lllll}
\hline Test & $\begin{array}{l}\text { CRH test }>430 \\
\text { nmol/L vs ITT }\end{array}$ & $\begin{array}{l}\text { Basal cortisol }>220 \\
\text { nmol/L vs ITT }\end{array}$ & $\begin{array}{l}\text { CRH test }>430 \mathrm{nmol} / \mathrm{L} \\
\text { vs all tests }\end{array}$ & $\begin{array}{l}\text { Basal cortisol }>220 \mathrm{nmol} / \mathrm{L} \\
\text { vs all tests }\end{array}$ \\
\hline Sensitivity & 0.556 & 0.556 & 0.742 & 0.842 \\
Specificity & 0.865 & 0.870 & 0.917 & 0.916 \\
Positive predictive value & 0.417 & 0.417 & 0.719 & 0.762 \\
Negative predictive value & 0.918 & 0.922 & 0.926 & 0.947 \\
Positive likelihood ratio & 4.119 & 4.277 & 8.940 & 10.02 \\
Negative likelihood ratio & 0.513 & 0.510 & 0.281 & 0.172 \\
ROC analysis AUC & 0.767 & 0.767 & 0.885 & 0.928 \\
Youden-index of cut-off & 0.421 & 0.426 & 0.659 & 0.758 \\
\hline
\end{tabular}

$C R H$ corticotropic releasing hormone, ITT insulin tolerance test, $R O C$ receiver operator curve, $A U C$ area under the curve

cut-off of $82 \mathrm{nmol} / \mathrm{L}$ corresponded with $100 \%$ specificity and a sensitivity of $22.2 \%$. The optimal cortisol cut-off value was $218 \mathrm{nmol} / \mathrm{L}$ or $325 \mathrm{nmol} / \mathrm{L}$, with a Youden-index of 0.426 (sensitivity $55.6 \%$ and specificity $87.0 \%$, and sensitivity $100 \%$ and specificity $42.6 \%$, respectively).

A ROC analysis with all different confirmation tests $(n=156)$ as reference yielded nearly the same cutoffs with higher statistical performances, the higher cutoff in this analysis being $411 \mathrm{nmol} / \mathrm{L}$, the lower $86 \mathrm{nmol} / \mathrm{L}$, and the optimal cutoff $218 \mathrm{nmol} / \mathrm{L}$, respectively.

Therefore, a cortisol concentration of $80 \mathrm{nmol} / \mathrm{L}$ (circa 86) was taken as the lower cutoff, $325 \mathrm{nmol} / \mathrm{L}$ as the higher cutoff, and $220 \mathrm{nmol} / \mathrm{L}$ as the optimal cut-off value. Performance indices for both references are presented in Table 4.

\section{Early postoperative basal (nonstimulated) cortisol versus confirmation test}

Mean postoperative basal cortisol concentrations in 156 patients were $321 \mathrm{nmol} / \mathrm{L}$ (range $9-909 \mathrm{nmol} / \mathrm{L}$ ).

Sixteen patients had cortisol concentrations below $80 \mathrm{nmol} / \mathrm{L}$. AI was confirmed during follow-up in all these patients (ITT $n=2$, CRH test $n=3$, ACTH test $n=1$, basal cortisol $n=10$ ). Postoperative basal cortisol was between 80 and $220 \mathrm{nmol} / \mathrm{L}$ in 26 patients, of whom 16 patients were diagnosed with AI during follow-up (ITT $n=3$, CRH test $n=9$, ACTH test $n=1$, basal cortisol $n=3$ ), and the other ten patients (38.5\%) had normal adrenal function (ITT $n=$ 7, CRH test $n=3$ ) (Table 2, patients 7-16). Therefore, 24\% of patients (10/42) with basal cortisol concentrations below $220 \mathrm{nmol} / \mathrm{L}$ had no AI during follow-up.

Postoperative basal cortisol was between 220 and $325 \mathrm{nmol} / \mathrm{L}$ in 39 patients. Of these, four patients $(10 \%)$ were diagnosed with AI during follow-up (ITT $n=4$, Table 3: patients 1-4). Thirty-five patients (90\%) had normal adrenal function during follow-up (ITT $n=22$, CRH test $n=13$ ). Postoperative basal cortisol was above
$325 \mathrm{nmol} / \mathrm{L}$ in 74 patients, and normal adrenal function was confirmed during follow-up in 72 patients (97\%) (ITT $n=24$, CRH test $n=23$, basal cortisol $n=25$ ). Two patients $(3 \%)$ were diagnosed with AI during follow-up (CRH test $n=1$, basal cortisol $n=1$, Table 3: patients 5 and 6). One of these patients was tested during hypothyroidism, the other during an episode of SIADH. Both presented with complaints of AI, one with hyponatremia, the other with severe fatigue. Hence, only 6 out of 114 patients $(5.3 \%)$ with early postoperative basal cortisol level above 220 were diagnosed with AI during follow-up. Performance indices of basal cortisol are presented in Table 4 .

\section{Incidence of adrenal crisis}

A total of six adrenal crises (grade $1(n=2)$, and grade 2 $(n=4))$ occurred in four patients, during a mean follow-up of 4.2 years (range $0.7-8.6$ years), and with a total of 656 patient-years of follow-up for the entire cohort. Three of these four patients had all been correctly identified after surgery as adrenal insufficient, and accordingly were put on hydrocortisone replacement. Adrenal crises occurred at 18, $28,35,54,61$, and 64 months, respectively, after surgery. One patient initially had been classified as false-negative based on the first postsurgical assessment, but this patient was correctly diagnosed with AI 6 months after surgery and was on hydrocortisone replacement thereafter (adrenal crisis occurred 54 months after surgery) (Table 3, patient 5).

\section{Discussion}

In this study, the diagnostic performance of a single basal cortisol measurement proved to be superior to the CRH test for the assessment of postoperative adrenal function (see Table 4). We found that the performance of basal cortisol measurements was sufficient to guide the postoperative hydrocortisone replacement scheme, that such a strategy 
was safe, and did not result in any case of adrenal crises in potentially misclassified patients. However, there were discrepancies between the early postoperative basal cortisol and the confirmation test during follow-up.

There are no studies available that compared the CRH test and basal cortisol in the postoperative setting. In the (nonpostoperative) diagnostic setting, Dullaart et al. reported that basal cortisol was not inferior to the CRH test [16]. Schmidt et al. even advised against using the CRH test because of its low sensitivity [14]. In our study, both tests misclassified a small number of patients (false-negative test results: $n=6,3.8 \%$ ). Another two patients had falsenegative results in the CRH test only (Table 3, patients 7 and 8). False-negative test results are of major concern in the assessment of adrenal function since untreated AI can potentially be life-threatening. As can be appreciated from Table 3, peak cortisol concentrations during dynamic confirmation tests were subnormal (between 328 and $424 \mathrm{nmol} /$ L) in four of these eight patients, and very low (between 11 and $51 \mathrm{nmol} / \mathrm{L}$ ) in the other four patients. The latter four patients are specifically intriguing, because of the large discrepancies between the early postoperative test results, and the test results during follow-up that can be explained by the following: first by the presence of untreated thyrotroph or somatotroph deficiency negatively affecting the cortisol response to stimulation. Thyroid hormone accelerates the endogenous clearance of cortisol [17], whereas growth hormone inhibits the conversion of cortisone to cortisol [18-21]. Consequently, the initiation or dose escalation of both thyroid hormone and growth hormone replacement can unmask impaired cortisol secretion. In agreement, two of these four patients had acquired secondary, and yet untreated, hypothyroidism after surgery (Table 3, patients 7 and 8), whereas the other two patients were already treated for preoperative secondary hypothyroidism (Table 3, patients 5 and 6). Three of the incorrectly classified patients had growth hormone deficiency. Another possible explanation is a subacute onset of AI in between the early postoperative evaluation and the second test, for example, due to late (pituitary) infarction. Finally, five of eight patients with false-negatives were tested during an (impending) episode of SIADH, or on irregular doses of desmopressin for DI. This may be explained by the costimulatory effect of vasopressin on ACTH secretion [22]. Of note, five of the eight patients reported severe fatigue prior to the diagnosis of AI

Vice versa, some discrepant test results in the group diagnosed with AI directly after surgery but with normal adrenal function during follow-up $(n=16)$ could be explained by late restoration of corticotroph function, or, although less likely, some suppression of corticotroph function due to the perioperative hydrocortisone replacement. In accordance, Pofi et al. recently demonstrated that restoration of adrenal function can occur even up to 12 months after surgery [23].

In the original assessment of the $\mathrm{CRH}$ test, the peak ACTH levels were incorporated. During this study, the peak ACTH levels altered none of the test results. An ROC analysis revealed an AUC of 0.673 (95\%CI 0.551-796) versus all confirmation tests and of 0.639 (95\%CI: 0.432-0.846) versus ITT alone. As the peak ACTH levels had no additional value for the test results, we omitted these data.

In a previous study, we evaluated the clinical applicability of postoperative CRH testing in our cohort treated from 1990 to 2009 [4]. The performance indices for the $\mathrm{CRH}$ test in that study were slightly lower than in the present study (sensitivity 67\%, specificity $87 \%$, PPV $69 \%$, and NPV 86\%). This can be explained, at least in part, by differing perioperative steroid replacement schemes, as dexamethasone was used in a majority of patients from 1990 to 2009 [4].

Studies have proposed different optimal cut-off values for early postoperative basal cortisol [14, 16]. The lower cut-off value obtained in this study is in agreement with already published data: $80 \mathrm{nmol} / \mathrm{L}(100 \mathrm{nmol} / \mathrm{L}$ with the cortisol I reagent) [12, 24, 25]. An exception is a study reported by Karaca et al., that suggested $129 \mathrm{nmol} / \mathrm{L}$ (165 nmol/L with cortisol I reagent) [10]. The two patients in our cohort with basal cortisol between 80 and $129 \mathrm{nmol} / \mathrm{L}$ had normal adrenal function during follow-up. The cut-off value for initiation of hydrocortisone replacement also differs between studies, with studies suggesting 234 [9], 312 [7] and 390 or between 299 and $390 \mathrm{nmol} / \mathrm{L}$ for clinically selected cases [8] (300, 400, 500, and between 370 and $500 \mathrm{nmol} / \mathrm{L}$ with cortisol I reagent, respectively). These discrepancies can be explained, at least in part, by differences between assays used, by different postoperative days of evaluation, by different perioperative glucocorticoid schemes, and by differences in treatment of other pituitary deficiencies. Therefore, centers should critically evaluate their own treatment and test results, and adjust cut-off values accordingly when implementing such a strategy.

A recent study by English et al. evaluated the accuracy of different tests for the assessment of postoperative adrenal function. They concluded that basal cortisol had a better diagnostic performance than a postoperative overnight metyrapone-suppression test. They also evaluated other tests and timing of the evaluation and concluded that a reliable formal assessment is only possible from 6 weeks postoperative onwards [7].

Limitations of this study are the retrospective evaluation and the fact that not all confirmatory tests during follow-up were done using the ITT, which is still considered the gold standard. As earlier stated, Schmidt et al. advised against using the $\mathrm{CRH}$ test because of its low specificity with the 


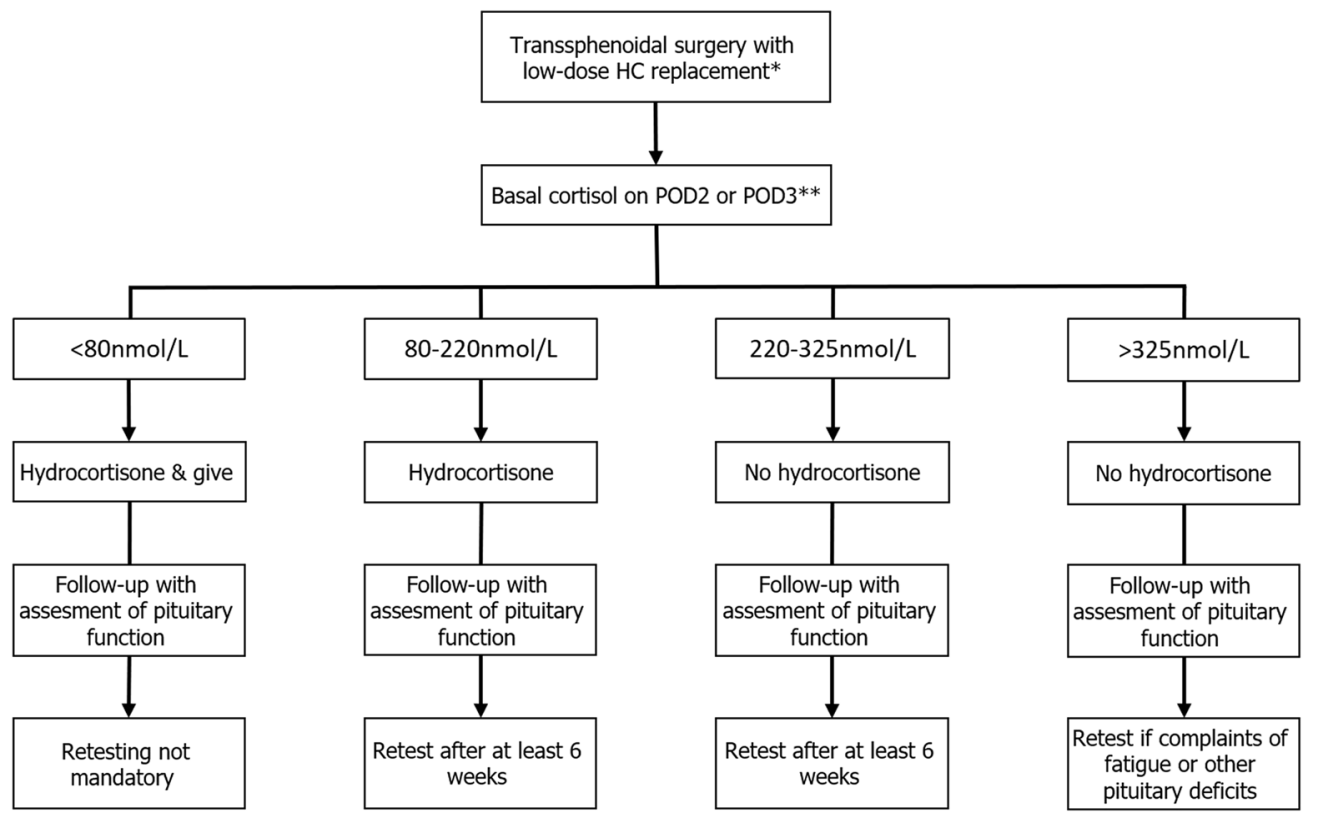

Fig. 3 Proposed treatment regimen for postoperative testing. HC: hydrocortisone POD postoperative day, Asterisk indicates $50 \mathrm{mg}$ of hydrocortisone $/ 24 \mathrm{~h}$ on the day of operation, tapered to $10-5-5 \mathrm{mg}$ of

cutoff we used. However, the test results of all patients that underwent another stimulation test (CRH test or ACTH test) were concordant and also in agreement with clinical symptoms. Also, the reagent for the cortisol assay was changed during the study period. This did, however, not significantly affect outcomes as mean basal cortisol levels were not significantly different, 317 vs $300 \mathrm{nmol} / \mathrm{L}$ ( $p=$ 0.556) before and after the introduction of the new assay reagent. In addition, peak ACTH levels during CRH test, which assay was not altered during the study period, were also somewhat lower in the group analyzed after the cortisol assay change (mean ACTH 88 vs $70 \mathrm{ng} / \mathrm{L}$ ).

In conclusion, the diagnostic performance of basal cortisol is sufficient to guide a postoperative hydrocortisone replacement scheme, and such a strategy is safe. The CRH test has no added value in this setting. Single, basal cortisol concentrations are easier to perform, more convenient for patients, and less costly. Consequently, we propose a new protocol for the assessment of adrenal function following pituitary tumor surgery (Fig. 3). Early morning basal cortisol samples should be obtained on the second or third day after surgery after withholding at least the evening and early morning dose of hydrocortisone. When basal cortisol is below $220 \mathrm{nmol} / \mathrm{L}$ hydrocortisone replacement should be continued. A formal assessment in those with postoperative basal cortisol between 80 and $325 \mathrm{nmol} / \mathrm{L}$ or symptoms suggestive of AI is advised at least 6 weeks after surgery. Reliability of testing can be optimized when testing is postponed in case of concomitant factors that can potentially affect $\mathrm{ACTH}$ and cortisol secretion and/or its hydrocortisone on postoperative day 2, Double asterisk indicates If the patient has no diabetes insipidus, SIADH, CSF-leak or fever

bioavailability, i.e diabetes insipidus, SIADH, CSF-leakage, fever, and oral contraceptive use in the 6 weeks before testing [26].

\section{Compliance with ethical standards}

Conflict of interest The authors declare that they have no conflict of interest.

Ethical approval All procedures performed in studies involving human participants were in accordance with the ethical standards of the institutional and/or national research committee and with the 1964 Helsinki declaration and its later amendments or comparable ethical standards. This study was approved by the Medical Ethical Committee of the Leiden University Medical Center. A waiver of medical ethical review was approved by our institutional medical ethical review board (G19.011).

Publisher's note Springer Nature remains neutral with regard to jurisdictional claims in published maps and institutional affiliations.

Open Access This article is distributed under the terms of the Creative Commons Attribution 4.0 International License (http://crea tivecommons.org/licenses/by/4.0/), which permits unrestricted use, distribution, and reproduction in any medium, provided you give appropriate credit to the original author(s) and the source, provide a link to the Creative Commons license, and indicate if changes were made.

\section{References}

1. M.A. Arraez, Assessment of postoperative hypocortisolism after pituitary surgery: when and how? World Neurosurg. 80, 495-497 (2013) 
2. V. Cerina, I. Kruljac, J.M. Radosevic, L.S. Kirigin, D. Stipic, H.I. Pecina, M. Vrkljan, Diagnostic accuracy of perioperative measurement of basal anterior pituitary and target gland hormones in predicting adrenal insufficiency after pituitary surgery. Medicine 95, e2898 (2016)

3. M. Klose, M. Lange, M. Kosteljanetz, L. Poulsgaard, U. FeldtRasmussen, Adrenocortical insufficiency after pituitary surgery: an audit of the reliability of the conventional short synacthen test. Clin. Endocrinol. 63, 499-505 (2005)

4. N.E. Kokshoorn, J.A. Romijn, F. Roelfsema, A.H. Rambach, J.W. Smit, N.R. Biermasz, A.M. Pereira, The use of an early postoperative CRH test to assess adrenal function after transsphenoidal surgery for pituitary adenomas. Pituitary 15, 436-444 (2012)

5. G. Zada, A. Tirosh, A.P. Huang, E.R. Laws, W.W. Woodmansee, The postoperative cortisol stress response following transsphenoidal pituitary surgery: a potential screening method for assessing preserved pituitary function. Pituitary 16, 319-325 (2013)

6. C.H. Courtney, A.S. McAllister, D.R. McCance, P.M. Bell, D.R. Hadden, H. Leslie, B. Sheridan, A.B. Atkinson, Comparison of one week $0900 \mathrm{~h}$ serum cortisol, low and standard dose synacthen tests with a 4 to 6 week insulin hypoglycaemia test after pituitary surgery in assessing HPA axis. Clin. Endocrinol. 53, 431-436 (2000)

7. K. English, W.J. Inder, Z. Weedon, G. Dimeski, J. Sorbello, A.W. Russell, E.L. Duncan, R. Cuneo, Prospective evaluation of a week one overnight metyrapone test with subsequent dynamic assessments of hypothalamic-pituitary-adrenal axis function after pituitary surgery. Clin. Endocrinol. 87, 35-43 (2017)

8. V. Hana, Jr. J. JeZkova, M. Kosak, M. Krsek, J. Marek, D. Netuka, M. Hill, V. Hana, Prediction of adrenocortical insufficiency after pituitary adenoma surgery using postoperative basal cortisol levels. Physiological Res. 64, 531-536 (2015)

9. C.N. Jayasena, K.A. Gadhvi, B. Gohel, N.M. Martin, N. Mendoza, K. Meeran, W.S. Dhillo, Day 5 morning serum cortisol predicts hypothalamic-pituitary-adrenal function after transsphenoidal surgery for pituitary tumors. Clin. Chem. 55, 972-977 (2009)

10. Z. Karaca, F. Tanriverdi, H. Atmaca, C. Gokce, G. Elbuken, A. Selcuklu, K. Unluhizarci, F. Kelestimur, Can basal cortisol measurement be an alternative to the insulin tolerance test in the assessment of the hypothalamic-pituitary-adrenal axis before and after pituitary surgery? Eur. J. Endocrinol. 163, 377-382 (2010)

11. N. McLaughlin, P. Cohan, P. Barnett, A. Eisenberg, C. Chaloner, D.F. Kelly, Early morning cortisol levels as predictors of shortterm and long-term adrenal function after endonasal transsphenoidal surgery for pituitary adenomas and Rathke's cleft cysts. World Neurosurg. 80, 569-575 (2013)

12. N.B. Watts, G.T. Tindall, Rapid assessment of corticotropin reserve after pituitary surgery. Jama 259, 708-711 (1988)

13. A.R. Hermus, G.F. Pieters, G.J. Pesman, T.J. Benraad, A.G. Smals, P.W. Kloppenborg, CRH as a diagnostic and heuristic tool in hypothalamic-pituitary diseases hormone and metabolic research. Suppl. Ser. 16, 68-73 (1987)

14. I.L. Schmidt, H. Lahner, K. Mann, S. Petersenn, Diagnosis of adrenal insufficiency: evaluation of the corticotropin-releasing hormone test and Basal serum cortisol in comparison to the insulin tolerance test in patients with hypothalamic-pituitary-adrenal disease. J. Clin. Endocrinol. Metabol. 88, 4193-4198 (2003)

15. B. Allolio, Extensive expertise in endocrinology. Adrenal crisis 172, R115 (2015)

16. R.P. Dullaart, S.H. Pasterkamp, J.A. Beentjes, W.J. Sluiter, Evaluation of adrenal function in patients with hypothalamic and pituitary disorders: comparison of serum cortisol, urinary free cortisol and the human-corticotrophin releasing hormone test with the insulin tolerance test. Clin. Endocrinol. 50, 465-471 (1999)

17. L. Persani, Clinical review: central hypothyroidism: pathogenic, diagnostic, and therapeutic challenges. J. Clin. Endocrinol. Metabol. 97, 3068-3078 (2012)

18. H. Filipsson, G. Johannsson, GH replacement in adults: interactions with other pituitary hormone deficiencies and replacement therapies. Eur. J. Endocrinol. 161(Suppl 1), S85-S95 (2009)

19. S.V. Gelding, N.F. Taylor, P.J. Wood, K. Noonan, J.U. Weaver, D.F. Wood, J.P. Monson, The effect of growth hormone replacement therapy on cortisol-cortisone interconversion in hypopituitary adults: evidence for growth hormone modulation of extrarenal 11 beta-hydroxysteroid dehydrogenase activity. Clin. Endocrinol. 48, 153-162 (1998)

20. C. Giavoli, R. Libe, S. Corbetta, E. Ferrante, A. Lania, M. Arosio, A. Spada, P. Beck-Peccoz, Effect of recombinant human growth hormone $(\mathrm{GH})$ replacement on the hypothalamic-pituitary-adrenal axis in adult GH-deficient patients. J. Clin. Endocrinol. Metabol. 89, 5397-5401 (2004)

21. P.M. Stewart, A.A. Toogood, J.W. Tomlinson, Growth hormone, insulin-like growth factor-I and the cortisol-cortisone shuttle. Horm. Res. 56(Suppl 1), 1-6 (2001)

22. R.A. Salata, D.B. Jarrett, J.G. Verbalis, A.G. Robinson, Vasopressin stimulation of adrenocorticotropin hormone $(\mathrm{ACTH})$ in humans. In vivo bioassay of corticotropin-releasing factor (CRF) which provides evidence for CRF mediation of the diurnal rhythm of ACTH. J. Clin. Investig. 81, 766-774 (1988)

23. R. Pofi, S. Gunatilake, V. Macgregor, B. Shine, R. Joseph, A.B. Grossman, A.M. Isidori, S. Cudlip, B. Jafar-Mohammadi, J.W. Tomlinson, A. Pal, Recovery of the hypothalamo-pituitaryadrenal axis following trans-sphenoidal adenomectomy for nonACTH secreting macroadenomas. J. Clin. Endocrinol Metabol. (2019). https://doi.org/10.1210/jc.2019-00406. [Epub ahead of print]

24. W.J. Inder, P.J. Hunt, Glucocorticoid replacement in pituitary surgery: guidelines for perioperative assessment and management. J. Clin. Endocrinol. Metabol. 87, 2745-2750 (2002)

25. N.F. Marko, A.H. Hamrahian, R.J. Weil, Immediate postoperative cortisol levels accurately predict postoperative hypothalamicpituitary-adrenal axis function after transsphenoidal surgery for pituitary tumors. Pituitary 13, 249-255 (2010)

26. E. Barel, R. Abu-Shkara, R. Colodner, R. Masalha, L. Mahagna, O.C. Zemel, A. Cohen, Gonadal hormones modulate the HPAaxis and the SNS in response to psychosocial stress. J. Neurosci. Res. 96, 1388-1397 (2018) 